\title{
Zum Nasaleinschub.
}

Aus dem Serbischen Ragusas werden čmbo, čobo (Zore Dubrovačke tugjinke S. 24 verzeichnet noch mit ital. Endung: combulin) in dem Sinne von "Mann" (in vertraulicher Rede), "Mandl" als Verkleinerungs- oder Koseformen von covek neben dem weiter verbreiteten covo angeführt. So u. a. von M. Rešetar Der štokavische Dialekt Sp. 153. 231 (an ersterer Stelle ist cobo irrtümlicherweise mit einem Stern versehen). Ich glaube hingegen dafs wir in den beiden ersten Formen das ital. ciompo „Knote“ (Mann von gemeinem Stande), sien. cigbo "Memme" o. ä., istr. tyo"bo "feister Mann" (Zeitschr. '07, I ff.) wiederzufinden haben; diese oder ihresgleichen sind mit dem slaw. čovo zusammengestofsen, haben ihm ihre Form aufgedrängt oder seine Bedeutung angenommen. $\mathrm{Zu}$ dieser Ansicht bestimmt mich auch die Schwierigkeit welche der Einschub des $m$ in einem so alltäglichen Worte bereitet. Zwar gibt Rešetar eine Reihe von slaw. Beispielen für die Entwicklung eines Nasals vor einem labialen Verschlufslaut (sowie vor einem dentalen) und eine noch längere K. Strekelj Zur slavischen Lehnwörterkunde S. 11 und 79 (auch von soichen für $n$ vor Guttural; S. 9. 15. 78 für $n$ vor Dental), und letzterer erkennt in den heutigen slawischen wie in den romanischen Sprachen "die Neigung, in fremden und einheimischen Wörtern, deren Stamm verdunkelt ist, vor $b, p$ ein parasitisches $m$ einzufügen" (S. II). Richtig erklärt werde dies von W. Foerster Zeitschr. '98, 264. Wenn man aber dessen Erklärung näher ansieht, so zeigt sie sich als eine lautphysiologisch genaue Beschreibung der neuen Aussprache wie sie von dem Ergebnis eines jeden Lautwandels geliefert werden kann. Das Ursächliche wird mit dem Hinweis auf einen "Sprachfehler" erledigt, „,indem, während der Lippenverschlufs des $b$ gebildet wird, durch Muskelschwäche oder sonst einen Vorgang das Gaumensegel herabfällt" setzt hinzu, er "kenne Individuen, deren Gaumensegel so schlapp ist, dals es fast bei sämtlichen Artikulationen herunterfällt". Diese Beobachtung ist zutreffend, enthält aber in sich selbst einen Einspruch gegen die Anwendung auf unsern Fall. Ein solcher pathologischer Sprachfehler, Kufsmauls Rhinolalia aperta, und ebenso ein entsprechender auf affektierter Lässigkeit beruhender (das aristokratische Näseln) offenbaren sich allgemein, nicht unter gewissen 
Bedingungen, am allerwenigsten unter den vorliegenden. Als eine schlaffe Aussprache von $a b a$ (entspr. ada, aga) ergibt sich $a m a$ (in noch höherem Malse $a m a)$, nicht $a m b a$; es gehört viel mehr Energie dazu das (iaumensegel wieder an die Hinterwand anzudrücken als es überhaupt nicht fallen $z u$ lassen. Und so findet sich denn $m b$ gerade für ein doppeltes, also energisches $b$ (z. B. neap. embe \} ebbene). Noch schwerer fügt sich der Einschub eines Nasals vor Tenuis in die Foerstersche Auffassung, am schwersten der vor Reibelaut. Ich schalte hier gleich, um keine Unklarheit aufkommen zu lassen, den positiven Ausdruck meiner Ansicht ein. Der die Media kennzeichnende Blählaut kann durch einen Nasal ersetzt werden, besonders wenn er gedehnt, aber auch wenn er einfach ist; ebenso die erste Hälfte der gedoppelten Tenuis, die Implosiva, wobei es sich genau genommen um die Ausfüllung einer Lücke handelt. Die Übergänge $a b b a\} a m l a, a b a\left\{a m b a, a p p a\left(a^{\prime} p a\right)\right\} a m p a$ nehmen in ihrer Folge an Wahrscheinlichkeit ab. Ob sie überhaupt selbständig vorkommen oder etwa nur im Zusammenhang mit andern Antrieben zur Nasalierung, das kann erst die Untersuchung der einzelnen Fälle entscheiden. - Foerster schlielst seine allgemeine Erörterung mit den Worten: „Dafs die Zahl der Fälle keine grofse ist, beweist, dafs alle die zahllosen Anläufe oder Ansätze durch spätere Korrektur (Schrift- und Gebildetensprache) ausgemerzt worden sind." Dieser Folgerung kann er selbst allerdings nicht ausweichen; ich aber ziehe aus jener Tatsache eine andere. Ich sehe nicht - ich bleibe hier in der gewöhnlichen Terminologie - ein "Lautgesetz" vor mir dessen Wirkungen bis auf verschwindende Ausnahmen wieder ausgelöscht wären, sondern einen „sporadischen“ Lautwandel und suche somit nicht nach einer allgemeinen Ursache, wie es Foerster tut, sondern nach besondern Ursachen. Wiederum verfährt er ganz folgerichtig wenn er (a. a. O. S. 509) in *gombitus) ital. gomito keine Anlehnung an cumbere einräumen will. Denn: "woran soll man die andern Fälle anlehnen? Hier ist eine Erklärung nötig, die alle Fälle einheitlich erklärt.“ Auch Štrekelj hält an dieser Einheitlichkeit fest: "Angesichts einer solchen Reihe von Fällen mit $m$ vor $b$, $p$ kann daher auch nicht recht die Rede von einer Anlehnung des cyborjum an cymbal [in cymborjum] sein" (S. 11). Aber er mildert dies durch das Wörtchen "recht", wie anderseits durch das Wörtchen "wohl" das Zugeständnis: "Das Wort oktumbar verdankt indes sein $m$ wohl den drei andern letzten Jahresmonaten auf -mbar" (S. 79). Diese seit lange anerkannte Angleichung ist uralt: schon zu Beginn des Mittelalters finden wir Beispiele von Octember und im Slawischen octomber nicht blols zu Ragusa, sondern auch anderswo, daher rum. octomvrie (s. Vok. des Vlgl. I, 38. III, I 2. 129). Übrigens ist Štrekelj auch bei früheren Gelegenheiten halben $\mathrm{Zu}$ geständnissen in derselben Richtung nicht ausgewichen. Von jeher hat er einen schlechtweg "parasitischen" Nasal anerkannt, zuerst allerdings in beschränktem Umfang: „Es zeigt sich das Bestreben in Wörtern, die mit ce, $c i$ anlauten und auf welche dann eine mit 
$p, b$ beginnende Silbe folgt, vor diese Labiale $m$ einzuschieben" (Arch. f. slav. Phil. '88, 461). Schon ebend. '92, 546 bringt er für den „nicht gar so unerhörten“ Einschub des Nasals andersgeartete Belege. In seinem Prinos $\mathrm{k}$ pornavanju tujih besed $\mathrm{v}$ slovenšcini, SA. aus Letop. Slov. Mat. '96, S. I 3. 16. 30. 3 I verweist er darauf dafs öfter $n$ vor $g$ und vor Labialen eingeschaltet werde. Anderseits aber bemerkt er ebend. S. 25 bezüglich des serb. plundre, neben poln. pludry "Pluderhosen": „Es ist schwierig zu entscheiden ob nicht vielleicht unsere Form, wegen des $n$, sich mit d. Plunder vermengt hat." Ebend. S. 30: "In tempast [„dumm" zu tip „Tölpel"] ist das $m$ eingeschoben wie in lampa, stembih, vielleicht unter dem Einflufs von tumpec, tumpast ["dumm"]." S. 31 wird serb. trancišek .,Mundkügelchen" ? ital. Irocisco + östr. Franzishierl (das gleiche, volksetymologisch umgebildete Wort) gesetzt. Schon früher, Arch. '92, 555, erklärt er das obersorb. zumpa ohne irgendwelches Bedenken ,aus d. Suppe mit Anlehnung an zump-Sumpf". Und an der allerersten Stelle meint er bezüglich des $m$ von cempin, cimbor, cimpreš (s. unten): „Die Einschaltung dieses $m$ kann bewirkt worden sein durch die Analogie der unter den Slovenen weit verbreiteten Wörter cimber, cimprman, cimprati...". Nun bieten aber eine Menge anderer Fälle ebenso unabweisbare Deutungen dar, und an sie wiederum lassen sich ohne Zwang weniger sichere Fälle anknüpfen. Ich beschränke mich im folgenden fast ganz auf den Stoff den Strekelj in dem angegebenen Sinne vorlegt; dazu kommen ein paar von Rešetar angeführte Wörter, und füge ich auf eigene Hand ein paar weitere, die ich durch gesperrten Druck auszeichne, von denen ich also nicht weifs wie Strekelj darüber denkt. Der Nasaleinschub im Slawischen geht auf drei Hauptquellen zurück, die zum Teil noch jenseits der Grenze entspringen (daher Štrekelj S. II: „es herrscht nämlich jetzt auch in den slavischen Sprachen ...6), und jedenfalls tritt er vor allem in Lehnwörtern auf.

1. Im Latein gibt es Stämme mit und ohne Nasal, z. B. accumliere: accubui, accubare; jungo : jugum. Indem die ursprüngliche funktionelle Bedeutung des Nasals nicht mehr gefühlt wurde, erschienen cumb- und cub-, jung- und jug-als lautliche Varianten und so wurden in früher oder später Zeit ein accumbitorium neben accubitorium, ein jungulum neben jugulum möglich. Für die romanischen Formen cumbet usw. durfte ich daher Vok. d. Vlgl. III, 93 f. ansetzen ein ,volkstümliches *cumbitus, das sich an *cumbere (in accumbere usw. erhalten) anschlofs", wenn mir auch diese Deutung trotz erhaltener Zustimmung nicht mebr ganz sicher ist (s. unten S. 88). Im Romanischen entstanden aber auch Dubletten rein lautlicher Art, indem hier ein alter Nasal blieb, dort schwand. So liefse sich rag.-serb. spendza "Aufwand" (R.) als eine Verschmelzung von ital. spesa mit einem neu belebten lat. expensa betrachten (wie ja auch wir Spesen und Expensen nebeneinander sagen); aber das $d z$ oder $z$ spricht eher dafür dals sich die sonstigen Formen des ital. Verbs spendere, spendo usw. ein- 
gemischt haben - verdankt doch auch franz. depense (: port. despesa) sein $n$ wohl eher dem Verb dependre als dem mlat. dispensa. Solche Formenmischung unterscheidet sich zwar nicht wesentlich von der unten $z \mathrm{u}$ besprechenden Wortmischung; während ich jedoch dann die direkte und einzelne Fortsetzung und Folge fremder Lautgebung im Auge habe, so jetzt nur die indirekte und allgemeine. Mit andern Worten: das Schwanken zwischen der Aussprache mit und der ohne Nasal welches in vielen fremden Wörtern aus gewissen Ursachen stattfindet, überträgt sich auf andere ins Slawische eintretende fremde Wörter und bereitet somit gleichsam den Boden für den Nasaleinschub vor, der sich dann unter bestimmten $\mathrm{Be}$ dingungen verwirklicht. Wichtiger noch dürfte sein dafs innerhalb des Slawischen selbst, gemäls dem Sprachcharakter, Entnasalierung an Lehnwörtern vorgenommen wird und dals daraus Fälle umgekehrter Aussprache erwachsen. In allgemeinen steht diese Tatsache gewils fest; aber unzweifelhafte Belege dafür beizubringen hält nicht ganz leicht. Wenn z. B. slow. žlambor "Erdeinsenkung", „Baumhöhlung“ (Štrekelj Slovarskí doneski iz živega jezika narodovega, SA. aus Letop. Mat. slov. '94, S. 60) auf friaul. slambra (vgl. auch ital. slamare) zurückgehen sollte, dann würde in der Nebenform žlabor das $m$ geschwunden sein; aber es gibt andere Möglichkeiten der Herleitung und nach ihnen würde die zweite Form die ältere, $m$ in der ersten also eingeschaltet sein. Sicher ist serb. slow. lubarda aus lumbarda, lombarda "Bombarde" entstanden; hat aber hier nicht serb. slow. $\}$ ital. alabarda eingewirkt, das ursprünglich auch $-m b$ hatte (mhd. helmbarte, oberl.-graub. halumbart, nizz. lambardo)? In slow. fródelj, serb. frúdalj "belaubte Zweige" ' friaul. frindej von frind, frund würste ich jedoch keine besondere Ursache für den Schwund des n.1 Endlich bleibt noch zu erwähnen dafs manche südslawischen 2 Formen von Erbwörtern einen Nasal enthalten von dem man zweifelhaft sein kann ob er primär oder sekundär ist. Štrekelj entscheidet sich grundsätzlich für das letztere. Wenn er sagt: „Ein dumbok [ ${ }^{\text {tief }}$ "] im Serbokroatischen ist meines Erachtens ebenso $z u$ beurteilen wie ein bumbrek, zumal jenes nie einen Nasalvokal hatte, wie ksl. dlübokü zeigt" (S. I I), so mag er Recht haben (wenngleich auf ksl. globokü zu verweisen wäre). Wenn aber E. Berneker Slav. Etym. Wtb. S. 215 mit Berufung auf Strekeljs Beispielsammlung in dem der čak. Md. Istriens angehörigen dumbrava "Wald" (R. führt es aus der štok. Md. der Bocche an) das $m$ als sekundär betrachtet, trotz ksl. dobrava, so wird es mir schwer in dem Zusammentreffen mit gleichbed. rum. dumbrava einen Zufall zu erblicken. Und man halte mir nicht slow. tump, tumpast neben top, topast $=$ rum. tîmp! ksl. top $\bar{u}$ nstumpf" entgegen; denn dort hat das $d$. Wort eingewirkt.

1 Vgl. slow. kolobar \} istr.-čak. kolombar ড̌trekelj S. 31, serb. buletati neben bulentin Zeitschr. '10, 736 .

2 Ich verstehe hier darunter nur das Slowenische und das Serbokroatische (oder kürzer Serbische). 
Fbenso liegt ja in kajk.-kroat. kundra „Zotte", kundrast ,kraushaarig", ung.-slow. kondrast .:zerzaust" $=$ slow. kodra, kodrast der Einfluls des madj. kondor "kraus", das selbst slawischer Herkunft ist, zu Tage, sodafs, wie Strekelj S. 35 sagt, on, un nicht etwa als erhaltener Nasalvokal zu betrachten ist. Aber wie ist slow. pomp-, pumpava ${ }^{1}$ neben pop-, pupava "Eberwurz" (auch tschech. pumpava neben pupaza) zu erklären, das zu pop, ksl. popic „Nabel", „Knospe“ gehört? In slow. ponglica neben poglica "Stecknadel", „Haftel" schien Štrekelj selbst (Doneski S. 35 f.) die Belehrung Levstiks dals das Wort für pongvica, ksl. pggrica stünde, auch in bezug auf das $n$ anzuerkennen.

2. Ein Nasal ruft in seiner Nachbarschaft vor einem Konsonanten cinen reuen Nasal hervor; er klingt vor oder nach. Dieser Vorgang lärst sich in seiner Wurzel von der Fernassimilation durch Nasal, vielfach auch in seiner äufsern Erscheinung nicht von ihr trennen. Z. B. * amendula amiddula kann so oder so aufgefalst werden (vgl. Meyer-Lübke Einf. ${ }^{2}$ 146). Wenn wir ein ameldula* hätten, so könnte man an ein aus $l$ assimiliertes $n$, aber auch an ein aus $n$ assimiliertes $l$ denken, endlich an selbständige Entwicklung von $l$ und von $n$ vor $d$ (vgl. Maldelena Salvioni Zeitschr. '98, 477 f.). Auch darüber wird man oft im ungewissen sein ob man von Fernassimilation reden darf; es kann ja ein Vokal + Nasal sich aus einem Nasalvokal entwickeln: *amêdula ; amendula. Wir haben nun, und zwar in Lehnwörtern:

a) den Nachklang eines Nasals. Der Vorgang hat sich schon aufserhalb des Slawischen vollzogen in poln. cmentarz ' mlat. coementerium usw. ' coemet., worüber jetzt Berneker a. a. O. S. 129 f., der auch hier wenigstens die Möglichkeit sekundärer Nasalierung zugibt. Seine Frage ob eine mlat. Form auf -arium zugrunde liege, ist $\mathrm{zu}$ bejahen, kommt doch in den Glossen ein mit coem. gleichbed. caementaria vor ${ }^{2}$ (im span. cimentero des Thes. ling. lat. - so MeyerLübke auch Rom.Gr.I $\$ 587$ - darf man keine Stütze für die Endung -arium suchen; span. heifst es cem-, cimenterio, wie a. a. $O$. $\$ 355$ ). - Serb. menǵurana "Majoran" (R.) finde ich in mittelgr. $\mu \alpha \gamma \gamma v \rho a ́ v \alpha$ (Langkavel Botanik der späteren Griechen S. 56) wieder; das $n$ könnte aus dem Romanischen stammen, doch vermag ich es aus einer naheliegenden Mundart nicht beizubringen; das Port. hat

1 Hieraus das ebenfalls slow. kompava durch Vermischung mit gleichbed. *kampana (kampaneža wird von Šulek bezeugt), welches seinerseits auf einer volksetymologischen Vermischung von lat. chamaeleon und spätlat. cardopanus (Th. 1. l. III, 447 oben), Bezeichnungen derselben Pflanze beruht. Aus einer umgekehrten Zusammensetzung dieser beiden: cardopanus und spätlat. camilina (Th. 1.1. III, 987, 4I) hat sich das mittelalterliche und von der Botanik adoptierte carlina ergeben, übersetzt Karlsdistel, kraljevac usw.

yas $n$ von coementerium kommt kaum auf Rechnung von caementum wie auch M. Vasmer Greko-Sl. èt. III, 222 anzunehmen scheint; vgl. mhd. dormenter \} dormitorium. In mhd. reventer (heute Remter), auf das mich Pogatscher aufmerksam macht, hat sich wohl allerdings ein anderes Wort eingemischt: refectorium, *revectorium + revenire. Vgl. unten S. 89 Anm. I. 
mangerona (die Stärkung der ersten Silbe scheint sonst lieber durch die Wiederholung des zweitfolgenden Konsonanten bewirkt worden zu sein: *marjorana, engl. marjoram, franz. marjolaine, wenn nicht in dem ersten $r$ ein Rest von amaracum stecken sollte). - Serb. Mandalena, Manda stammt aus ital. Mdd. (istr. Mandalena Ive S. 36. 114 ; altberg. Mandelena Arch. glott. ital. XIV, 233). - Nanga „Neigekommt ganz auf die Rechnung des Slowenischen. Ebenso trombenta ? ital. trompetta, das aber durch Anlehnung an troba $\}$ tromba wieder das gewöhnliche trobenta ergeben hat; vgl. mhd. trúmenter neben trúmeter. - Endlich ziehe ich hierher noch ein echt slowenisches Wort: das niémnı̌ki des Karstdialektes für němš̌ki (Śtrekelj S. 9 lärst das $n$ vor $\check{c}$ entstehen: *němnčski).

b) Der Vorklang eines Nasals ist im Romanischen nicht selten 1 und dürfte von da in einem oder dem andern Worte ins Slawische gekommen sein. Ganz sichere Beispiele aufser serb. angonija (Zore) ! alt- und mdl.-ital. (auch istr.) angonia sind mir nicht zur Hand. Das istr.-čak. kankîn "Stöpsel “ trägt Śtrekelj S. 25 Bedenken mit concon, cuncon von [Reggio und] Modena zusammenzubringen, da dieses etwas $z u$ entfernt sei; er denkt an ein aus friaul. chalcon $\mathrm{zu}$ erschliefsendes *calcon (vgl. neugr. xov@xovivı Som., märk. crocchione, neben cocchione, cuccone), zu welchem er Prinos S. 14 slow. kotkīn, (im Karst) kowkýn gestellt hatte. Aus DC. zitiert er cochonus; er hätte da auch conconus finden können, aus einer Chronik von Treviso geschöpft - über Weiteres habe ich mich nicht unterrichtet - , und Treviso ist für den besagten Zweck nahe genug. Überdies könnte ja der Nasaleinschub sehr wohl an verschiedenen Orten selbständig vollzogen worden sein. Das Gleiche gilt für serb. inkona, inkuna neben ikona \} mittelgr. cixóva "Heiligenbild" (Berneker Wtb. S. 424), welches sich mit ital. ancona "Heiligentafel“, „Heiligennische" begegnet, parm. auch incona, südital. cona. Innerhalb des Slawischen und zwar des Slow. haben sich sicher entwickelt cempin\} cepin, capin ${ }_{\text {"Hacke }}{ }^{\text {}}$ ital. zappino, čeronten ' ital. serotino (Arch. '92, 519 f.), london „Zürgelbaum " ? istr. - ital. lodogno (S. 36), istr. - čak. kontenina (slow. kortenina) „Baumwollenzeug“ 4 ital. cotonina (da dies Wort im Ital. besteht, so ist es nicht nötig mit Strekelj S. 33 den Antritt der slaw. Endung -ina an koton anzunehmen). Ganz so wie inkona zu ikona verhält sich ein Wort von slawischem Stamme:

1 Im Romanischen ist der Vorklang des Nasals oft verkannt worden und ist doch so leicht zu erkennen, besonders wenn er in Ableitungen auf -in, -on. -an erscheint, wo dann die Stammwörter als Gegenzeugen auftreten, z. B. altfranz. roncin : rosse, friaul. rinçhin (riçhin) : ital. orecchia, mdl. -ital. donzena : franz. douze. Dadurch werden wir befähigt dunkle Fälle aufzuhellen wie den von südital. langella für *lagella (d. Lägel); wir setzen ein *langena an und dieses wird bestätigt durch die alte Schreibung languena (Vok. d. Vlgl. II, 280; so ital. 1207 bei DC., welcher auch languella und franz. 1406, langeau bietet). Neben ital. marangone, parangone, Sansonia, istr. șanșein (assassino), BambiLonia u. a. war doch auch mod. sonzon \} *sozzone klar, wo Salvioni Zeitschr. '98, 477 Einmischung von sonsa \}axungia vermutet. 
serb. inglün zu iglün, nach Rešetar S. 238 „Art Seefisch", wohl dasselbe wie jaglun "gemeiner Schwertfisch" und "Segelfisch" (jagla ist die čak. Form von igla "Nadel"; vgl. jaglica, iglica "Hornhecht"). - Unter den deutschen Lehnwörtern befindet sich eines das im Deutschen selbst Lehnwort ist und schon hier den Nasaleinschub erfahren hat: Bajonett \{ mdl.-d. (wien. tyrol. bair. egerl. henneb. erfurt. niederrhein.; s. Schmeller B.Wtb. ${ }^{2}$ I, 250. Gartner Zeitschr. f. hochd. Mdd. IV, 275) Banganet, Bankenet u. ä., jüt. bangenet (Feilberg I, 4I), madj. panganét, manganét, pangallít 1 (Szinnyei M. Tájszótár II, 70. V.Lumtzer und J.Melich D. Ortsn. u.Lehnw. des ung. Sprachsch. S. 191), serb. panganet. Slow. štenge setzt Śtrekelj S. I I (Arch. '92, 546: im Mittelkarstdial. štényo) dem d. Stiegen gleich, also der alten Form, nicht dem heutigen östr. $\check{S}_{t i}{ }^{a} \dot{n}$; ebenso ist prunka (Arch. '92, 537) neben pruka "Betstuhl" auf östr. Brucken zu beziehen; der erregende nasale Auslaut ist geschwunden ähnlich wie in port. pente pentem $\}$ pectine. Klar ist slow. cvenčnik neben cvečnjak "Vorbohrer" von cvek? d. Zweck. Sehr schwierig ist das slow. spancirati "spazieren gehen"; da das auch spät entlehnte holl. spanseeren dazu stimmt, mufs die Ursache des $n$ im Deutschen gesucht werden. Ein sich spannen im Sinne von "sich brüsten" kommt in d. Mdd. vor, scheint aber wenig verbreitet zu sein; sonst würde es das $n$ in dem slow. wie holl. Verb erklären können, die beide insbesondere "einherstolzieren" bedeuten (umgekehrt slow. prangati "spazieren gehen" Strekelj S. 49). Vielleicht hat das $-n$ des Infinitivs gewirkt, der ja in seiner ständigen Verbindung mit gehen eine feste, sozusagen adverbiale Form angenommen hat. - In einigen Fällen Strekeljs ist das $n$ gar nicht eingeschaltet, sondern versetzt, so slow. salintra ital. salnitro, d. Salniter (aber salitra franz. span. salitre) und serb. ambis \} altital. nabisso, oder aus einem andern Konsonanten abgeändert: kajk.-kroat. cuntar \}ä.-madj. csoltár, csultár, rum. croltar \} türk. collar \{ serb. cuultan, nicht vom jüngeren madj. csótár "Schabracke". Vgl. hierzu das von Strekelj S. 79 besprochene slow. serb. mantrati neben martrati $\}$ d. martern (vgl. die Dissimilation in ä.-d. Martel und in d. Mörtel, mdl. Malter). Ferner tschech. Stencle \} d. Stelsen (Arch.' 92,546 ; nicht eher ' Stelzel?) und slow. candra usw. „Fetzen" $\}$ kärnt.-d. zalder "Faserichtes" (Arch. '06, 510). 2

3. Der Nasal wird aus einem andern Worte übertragen, und zwar um so leichter je gröfser die lautliche und begriffliche Ähnlichkeit ist. Manche der hieher bezogenen slawischen Wörter haben ihn aber schon aus der Fremde mitgebracht. An die Spitze eines Artikels der ausführlich die slawische Einschaltung eines Nasals behandelt, hätte ein anderes Wort gehört als tschech. cimbur, cim-

1 Man bemerke wie in der zweiten Form die Nasalierung sich rückwärts ausbreitet (Assimilation des $p$ ), in der dritten wicder nach vorn eingeschränkt wird (Dissimilation des ursprünglichen $n$ ).

2 Es ist hier an das ital. cialtrona zu erinnern, das auch umgekehrt aus *ciantrona entstanden sein könnte. 
bura, cimburi, (alt) cimbořie „Zinne“, „Mauerkranz", „Kranz am Ofen"! mhd. ziborje "Hostienkelch mit baldachinartigem Deckel“, „Säulenhäuschen", "baldachinartige Krönung" ' gr.-lat. ciborium, wozu sich poln. cymborjum neben cyborjum im eig. S. gesellt. Es entspricht nämlich mlat. cimborium, cymborium, cimbarium "Altarbaldachin", span. cimborio, cimborrio, (alt) cimorro, kat. cimbori, port. zimborio, -ria "Laterne oder Aufsatz einer Kuppel", „Kuppel" (vgl. lang. cibòri "Kirchentor"). dessen Beziehungen zu * cimarium „Helmwulst", span. cimbra, port. cimbre "Gewölbbogen", altspan. (gal.?) cimbro "Gipfel" ich hier nicht $z u$ untersuchen brauche. - Das $m$ des von Rešetar angeführten serb. limbijao neben lubijao "Seebarsch" gehört ebenso wie das des gleichbed. serb. lumbrak neben lebrak schon dem Romanischen an (s. Zeitschr. 'o7, 642 ff.). - Zu slow. špáranga aus ital. sparago setzt Strekelj zwar: "auch ngr. $\sigma \pi \alpha \rho \alpha ́ \gamma \gamma \iota^{\prime \prime}$; ist es aber nicht unmittelbar auf dieses zurückzuführen (Arch. '92, 546 sagt er, "es liege zu weit ab"), wie rum. sparangă, span.-(afr.-)arab. is farandža u. ä. (Simonet S. 192)? Gelegentlich des griech. Wortes sagt M. Vasmer Greko-Sl. èt. III, 189, Anm. I : „Von der sekundären Nasalisation im Griechischen Ausführlicheres noch an anderem Orte.“ Ich glaube hier handelt es sich um einen ganz besondern Fall:

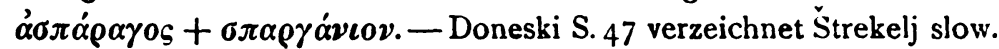
telengati "wackelnd einhergehen" und vermutet dessen Herkunft von telega "Schubkarren", dessen Rad gewöhnlich "wackle"; sollte das Verb nicht eigentlich soviel sein wie "einen Schubkarren führen" oder "auf einem Schubkarren fahren", sodafs es zu madj. taligázni stimmte? Über das $n$ äulsert er sich nicht; bulg. talingar führt er wohl ohne bestimmte Absicht an, sonst wäre noch, nach Miklosich, lit. talenge, tolenga zu nennen gewesen. Nun kommt aber aus Pleteršnik hinzu telenge $=$ telege (Pl.) "Ochsenjoch" (im Sing.: „der innere lange Spriefsel des Ochsenjoches"; in der Bed. "Karren" hat er diese Form nicht). $\mathrm{Da}$ der Ursprung des Wortes selbst noch im dunkeln liegt - der angenommene türkische scheint mir nicht ausgemacht -, so läfst sich auch über den des $n$ nichts feststellen. - Es kann vorkommen dafs der Vorgang sich zwar auf slawischem Boden abspielt, das Wort aber das den Nasal einem andern mitteilt, im Grunde noch aufsen steht. Strekeljs Behauptung dafs das $n$ in (ält.) slow. buntora, buntara nebén butora, butara 'madj. buitor

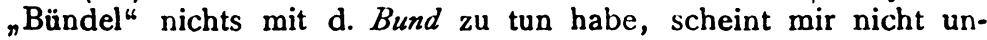
anfechtbar. An der madjarischen Sprachgrenze nahmen die Slowenen die auch der andern Sprache mehr oder weniger mächtig waren, das madjarische Wort auf und es bürgerte sich ein; an der deutschen Sprachgrenze brachten deutschkundige Slowenen es mit dem deutschen Wort in Verbindung und glichen es ihm an. Aber ich sehe im folgenden von diesem den Kern der Sache nicht berührenden Unterschiede zwischen fremdem Wort und Lehnwort ab und muls von ihm absehen, da er meistens schwer festzustellen ist. Ebenso mufs ich mich über ein anderes hinwegsetzen, obwohl es von gröfserer Bedeutung ist: die unnasalierte Form läfst sich nicht immer im 
Slaw. nachweisen und so ist die Möglichkeit gegeben dafs sie nur in der andern Sprache besteht; es könnte sogar das oben erwähnte plundre, trotz poln. pludry, direkt auf ein d. ${ }^{*}$ Plunderhosen zurückgehen. - Slow.buncelj "Nocke“' 'bair. Wutzel „Wulst", + slow.bunčica "Knöllchen", Dem. von bunka "Knorren" u. ä. ' bair. Pünkel, -en. - Slow. cimbor (a) neben cibora \} südd. Zipper u. ä. "Kriechenpflaume“" (prunus insititia) scheint sich irgendwo an friaul. cimbar "welsche Mispel" (crataegus azarolus) angelehnt zu haben. Strekelj Arch.'9o, 482 nimmt umgekehrten Zusammenhang an. - Ähnlich serb. cimpres, cempres, cimpres, cempres, cempris neben cipres, cepris, cepris u. ä. "Zypresse" , slow. cemprin "Zirbelkiefer" (vgl. d.Zirm, Zember, graub.lad. (d)schember). - Slow. gombela „Bogen über der Wiege" neben serb. gobel(j) $a$ ! istr.-ital. gaveja, friaul. giavel, mail. gavell usw. "Radfelge“, + slow. kamba, kumba, cak. kamba "Bogen am Ochsenjoch", res.slow. kamba "Holzband der Viehglocke" i kelt.-lat. camba (port. camba "Radfelge"; s. Nigra Zeitschr. 'O3, 13 I f.). Strekelj hat sich über kamba Arch. '88, 46r. '91, 526 und über gobelja Zur sl. L. S. 20 und Arch. 'o6, 512 geäufsert; an dieser letzten Stelle scheint es dafs er gobelja, gaveja unmittelbar aus camba herleiten will. Hierbei hat er übrigens einen Ausdruck Ives, der allerdings nicht hinlänglich scharf geprägt war, milsverstanden; unter gavello falst ja auch Ascoli Arch. glott. ital. VII, 547 die betreffenden lad. und oberital. Formen zusammen, ohne damit etwas über den Ursprung $z u$ sagen. Serb. kalonperje neben kaloper "Frauenmünze" verhält sich wie serb. karanfil neben karavilje "Nelke"; beide sind nämlich im Grunde ein Wort: ${ }_{*}$ caryophyllum, welches zum Teil und zwar wenn für die Nelke eine andere Bezeichnung aufkam, auf die erstere Pflanze übertragen wurde, so noch russ. kalifer, kaniper. Für das $n$ vor $f, p$ ist wohl das Türkische verantwortlich zu machen (s. Miklosich Die türk. Elemente in den südosteur. u. osteur. Spr. S. 91). - Slow. klangovati neben klagovati "klagen" (Štrekelj Prinos S. I 3 führt die zweite Form nicht an, Pleteršnik nur sie), + klengetati, klenkati $\}$ bair. klenge(I)n. - Slow. lampa "Lippe“ neben lapa (bes. im Pl.) "Maul" \} bair. Lepo'n (Prinos S. 16), + slow. zlampati $\}$ d. schlampen (vgl. friaul. slapâ dass., "lecken" [von Hunden], lapa dass., usw. im Rom.). - Slow. plundra neben plodra "Patsche“, .Kotbrühe" imdl.-d. pludern ,im Wasser platschen" (hd. plaudern entspricht besser dem Laut, holl. ploeteren dem Sinne nach; vgl. auch bair. Schneegeplöder Schmeller 2I, 457), + slow. plunkati, pluncgati, plunkniti neben plukati, pluskati ähnlich wie d. p(l)antschen neben plätschern. - Slow. prungelj neben prugelj\} d. Prügel, + slow. bunkati "schlagen" ' mhd. punken (vgl. Strekelj S. 8) oder $+\mathrm{d}$. Bengel. - Slow. rándiga ' ven. radigo "Streithandel", + d. Randal. Strekelj hat Recht die Herleitung vom deutschen Wort (Arch. '92, 539) aufzugeben, Unrecht auf dieses ganz zu verzichten (Z. sl. L. S. 5I). - Slow. škindra "Splitter" für *šidra \} ital. *schidola (oder hätte das *skidva ergeben?) von bresc. crema. cremon. mant. schida dass. + istr.-̌̌ak. žlendra ' friaul. sclèndare (Štrekelj S. 76; vgl. ven. schienza : friaul. ital. scheggia; trev. vic. s'cianta : franz. éclat; ndd. 
Splinter : d. Splitter usw., worüber bei anderer Gelegenheit mehr). Man kann, vom Anlaut abgesehen, auch an bair. Flinder $=$ Flitter denken und zwar wie für škindra so für slow. cefindra \} cefedra "Splitterchen", „Fetzchen". - Slow. štembih, stempih neben stepih "Pumpbrunnen" \& bair. Stübich "Packfals" (Prinos S. 30), + bair. Stempen nkurzer Pflock“. - Slow. štu mbelj neben štubelj "länglicher Topf " ' d. Stübchen „ein Getränkemafs" (Prinos S. 30, wo aber die Form mit $m$ nicht erwähnt wird), + bair. Stampe, Stamperl „Trinkglas mit dickem Fufs". - Slow. tumpelj, tompelj\} d. Döbel, + tump ? slow. top, + d. stumpf; davon tompljati "s tomplji zbijati", welches Štrekelj S. I I aut d. doppeln bezieht. - Slow. žlundra neben zlodra, żloter "Kotsuppe", „Schlamm" \} d. Schlotler (Strekelj S. 14), + žlindra, żlintra, zlinter "Schlacke, Unreinigkeit" (\} d. Sinter + Schlacke) und wohl noch eher + gleichbed. plundra neben plodra (s. oben). - Der begriffliche Zusammenhang zwischen dem abändernden und dem abgeänderten Wort kann ein sehr loser sein. S. 3 I sagt Strekelj von istr.-čak. kombulica, das er als Deminutiv von ital. tombola ansieht: „Der Anlaut scheint mit Rücksicht auf die vielen ital. Komposita mit con- in kom- gewandelt worden zu sein." Er hätte diese Bemerkung auch für das bei ihm unmittelbar darauf besprochene rag.serb. kompric, komprę verwenden können, das er gewifs richtig dem ital. capriccio gleichsetzt. Wenn aber - wie er sagt - „das $o$ der ersten Silbe wohl kaum o, $u$ einer romanischen Sprache ist, da es in Ragusa $u$ ergeben würde", so wird dadurch auch die Einmischung von con- einigermalsen zweifelhaft gemacht (indessen finde ich in den Dubrovačke tugjinke von Zore komplita, konbenat neben kùmbulj). Vgl. auch slow. kumplati ' d. kuppeln (Strekelj S. 1 I). Noch leichter stehlen sich in Lehnwörter unbefugter Weise fremde Endungen ein; so erklärt sich z. B. slow. barantati neben baratati ${ }_{\text {Handel }}$ treiben" $\}$ ital. barattare "tauschen" leicht aus dem -ant- der Partizipien und davon gebildeten Ableitungen (z. B. commerciante, negosiante, mercante - mercantare). Ganz ebenso mndl.barenteren neben barleren $\}$ altfr. bareter "tauschen" (J. J. Salverda de Grave De Franse woorden in het Nederlands S. 347). Man vergleiche dazu mdl.-d. visentieren (schon früh-mndl. visenteren a. a. O. S. 290; altwall. visenteir Risop Jahresb. II, 170), profentieren, spedentieren u. ä. nach präsentieren, parlamentieren, lamentieren usw. Ähnlich zu erklären ist rag.-serb. oblanta

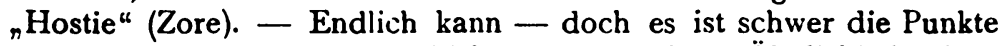
zu kennzeichnen wo es geschieht - die äufsere Ähnlichkeit eines Wortes mit einem andern genügen damit es ihm noch ähnlicher werde. Das aus dem Türkischen entlehnte serb. slow. bur, bobreg, $-k$ "Niere" erscheint auch als bum-, bombreg, $-k$ vielleicht nur im Anschlufs an die ganz anderes bedeutenden Wörter mit bumb-, bomb-. - In manchen der vorgeführten Beispiele wird man glauben das leise Rauschen noch einer weiteren Quelle zu vernehmen, der onomatopoetischen. Allein sie lärst sich nicht als eine besondere aufzählen, weil die Lautnachahmung fast immer schon aufserhalb des betreffenden Wortes in bestimmten Wortformen ausgeprägt ist. Wollten wir z. B. aus slow. 
cintre (nicht bei Pleteršnik; serb. cindra) neben citre $\mathrm{Pl}$. „Zither" den unmittelbaren Widerhall des Instrumentes heraushören (vgl. altfranz. guinterne, südfranz. guindarro, ginjarro, tintarro „Guitarre“), so könnte man uns auf slow. plinkati "klimpern", cinkati (auch serb.), cingati "klingeln", tintina „Maultrommel" u. a. verweisen. - Unter ganz denselben Umständen wie Lehnwörter, erfahren auch Erbwörter die Einschaltung eines Nasals, also wiederum sind Lehnwörter die wirkenden. Slow. cempèr neben ceper (zu cepiti "spalten") "Splitter", "Stückchen", + cembi „Fransen" 'madj. csembo" „Franse"? - Slow. cuncati neben cucati "schwerfällig gehen", + cincati dass., cencati "wackelnd einhergehen ", „,zaudern"! bair. zünzeln „sich langsam, bedächtig, verlegen benehmen", „zaudern". - Serb. (rag.) čumprelica, čumprela ,gemeines Mädchen “, Dem. von čupa ,Weib mit ungekämmtem Haar:" (s. Berneker Wtb. S. I60), + bair. Schummel, Schumpel (verächtlich) „Weibsperson" oder Schlump „unreinliche Weibsperson“ oder Fem. von dem eingangs erwähnten ital. ciompo. ${ }^{1}$ - Serb. klempati neben klepati ${ }^{2}$,dengeln", + d. klcmpern "hämmern". - Serb. kombacati se neben kobacati se "wit den Fülsen scharren", kompricati se neben kopricati se "zappeln" durch ital. com- beeinfluist? - Serb. prandid (R.) neben praded "Urgrolsvater", + čukunded „Ururgrolsvater". Ich sagte oben dafs ich des Wandels von gedehnter Media und gedoppelter Tenuis zu Nasal + Verschlufslaut als selbständigen Vorgangs überhaupt nicht sicher sei. Am wenigsten nun für das Slawische, dem zwar die Gemination fremd, aber auch die Verbindung Nasal + Konsonant fremdartig ist. Immerhin vermag ich in einzelnen Fällen, z. B. bei slow. žlemprha „Falltür" \} mhd. slegebrïcke (Iz besednega zaklada narodovega, SA. aus Letop. Mat. slov.'92, S. 47) keine andere denkbare Ursache zu entdecken ( $g p\{p p ; m p)$.

Es bleiben noch einige der von Strekelj angeführten Wörter übrig; ich könnte, wenngleich mit etwas grölserer Schwierigkeit, auch hier Erklärungen des eingeschalteten Nasals aufbringen. Aber vielleicht befriedigen schon meine bisherigen nicht, und ich steife mich selbst keineswegs darauf. Es sirid nur Lückenbüfser; möge er sie beseitigen oder berichtigen, er besitzt, was mir abgeht, die tiefe Kenntnis aller der Umstäncle die bei einer derartigen Forschung zu würdigen sind. Nur daran liegt mir dafs er das Vorhandensein der Lücken anerkenne und die Möglichkeit sie nach meiner Methode auszufüllen. Ich denke doch, ich habe das Gewebe fest angeknotet, flattern auch die Enden im Winde.

Von einem romanischen Flüchtling habe ich mich auf slawischen Boden verlocken lassen und hier an Strekeljs Hand einen längeren

I $\mathrm{Da}$ ciompo auch "Wollkratzer" bedeutet, so mag auf Wollschlumper "Art Arbeiter (Wollkratzer?)“" bei Schmeller ${ }^{2}$ II, 525 hingewiesen werden.

2 Wenn Budmani zu diesem Worte im Rjeǐnik V, 53 bemerkt dafs die Einschaltung des $m$ erst in neuerer Zeit erfolgt ist und ${ }^{\text {ne }}$ treba misliti da je ostatak praslavenski", so war hier der Gedanke an einen urslawischen Nasal (s. oben S. 74) von vornherein ausgeschlossen.

Zeitschr. f. $九 0 \mathrm{~m}$. Phil. XXXV. 
Spaziergang unternommen. Wenn er sich durch eine Ansicht beeinflussen liefs die an romanischem Stoff gewonnen war, so kann nun dessen Beurteilung wieder vom Slawischen her erleuchtet werden. Doch mufs ich das noch allgemeiner formeln. Ich bin durch Zufall an eine mir schon vertraute Stelle geraten: seit langer Zeit hege ich die Überzeugung dafs eine vergleichende Lautgeschichte die aufserhalb der gewöhnlichen Sprachvergleichung steht, oder mit andern Worten sich nicht auf die Verwandtschaft zwischen den Sprachen stützt, auch für die Forschung auf den Einzelgebieten nicht nur nützlich, sondern vielfach sogar notwendig sich erweist. Das letztere nun auch in unserem Fall; das Wesen des Nasaleinschubs läfst sich innerhalb enger Grenzen nicht wirklich ergründen. Gerade je verschiedenartiger die Grundlagen sind auf denen sich die im wesentlichen gleichen Vorgänge abspielen, desto stärker werden diese erhellt werden. Anstatt einer systematischen Darstellung, an die ich gleich von vornherein hätte denken müssen, möge man ein paar kleine Beiträge dazu sich gefallen lassen. Ich werfe zunächst einen raschen Blick auf das Englische, dann auf das Griechische und schliefse mit einigen allgemeinen Bemerkungen.

Das Englische und das Südslawische gehen in bezug auf Lautsystem, Betonungsverhältnisse, Wortbau und anderes möglichst weit auseinander, vor allem darin dals die Verbindung Nasal + Konsonant, von der neue Prägungen geschehen, dort eienso gewöhnlich ist wie hier ungewöhnlich. Gemeinsam aber ist beiden dals es gröfstenteils spät zugewanderte Wörter sind die den Nasaleinschub zeigen, ja dals er manchmal sich so zu sagen vor unsern Blicken $\mathrm{zu}$ vollziehen scheint. Ich kenne folgende Auslassungen über den englischen Nasaleinschub: O. Jespersen Engl. Stud. 31 ('O2), $239 \mathrm{ff}$,, H. Logeman ebd. 34 ('O4), 249 ff., O. Ritter Arch. f. d. St. d. n. Spr. I 13 ('04), 3 I ff., K. Luick ebd. I 14 ('o5), 76 ff., W. Horn Untersuchungen zur neuenglischen Lautgeschichte 'o5, S. 63 f., O. Jespersen A modern english grammar 'o9, $\$ 2.429$ und 2. 432. Wenn auch zwischen den Genannten keineswegs Einstimmigkeit herrscht, so schliefsen doch die von ihnen angenommenen Ursprünge einander nicht geradezu aus. Bei allen aber vermisse ich den Hinweis auf die Hauptquelle des sekundären $-n$ - oder $-\dot{n}-$, nämlich die Nachbarschaft eines Nasals. Ritter zählt 20 Belege (auch ein paar altenglische) für den Nasaleinschub vor $d$ und $t$ auf; von diesen enthalten 6:m-(n) (cumendre), 2:m--(n) (milintary), 7:(n)-n(skelinton); in einem Fall ist das (n) schon aufserenglisch; in den vier übrigen haben entweder diese Vorbilder nachgewirkt oder hat irgend ein anderes Wort sich eingemischt (brigander für brigadier). Was allerdings den Nasaleinschub vor $g^{\prime}(d \check{z})$ anlangt, so scheint die Statistik $\operatorname{mir} z u$ widersprechen; denn es liegen wenig solche Formen und wohl nur eine alte mit ursprünglichem Nasal vor: messenger \{ messager. Vielleicht ist das $n$ hier schon aufserenglisch; auch das frühe Mndl. hat messengier; ja man könnte sogar auf port. mensageiro, span. mensajero zurückgehen, woraus die andere Form durch Um- 
stellung des $n$ entstanden wäre. Dieses messenger aber zog passenger und die übrigen Wörter auf -(e-, a-, i)nger für -ger nach sich, die ja auch in nahem begrifflichen Zusammenhang zu ihm und untereinander standen (in manager unterblieb die Einschaltung des $n$ wegen des vorausgehenden $n$, wie Jespersen ${ }_{1}$ sagt); hier mufs man das Wägen an Stelle des Zählens setzen. St. Leger gespr. silindžd pafst freilich seiner Bedeutung nach nicht in diese Gefolgschaft; aber hier ist auch das $n$ nicht sowohl eingesprungen als (aus der ersten in die zweite Silbe) umgesprungen (das $\mathrm{Zu}$ sammentreffen mit mdl.-ital. lenger, linger - s. unten S. 91 - ist also zufällig). Dem messenger-Typus steht der nightingale-Typus zur Seite, der aber, da sich martingale und Portyngale als schon aufserenglisch erwiesen haben, sehr zusammengeschmolzen ist. Nightingale findet sich als altes volkstümliches Wort recht vereinsamt; auch ist hier der vorwärtswirkende Nasal nicht wie sonst ein $m$, sondern ein $n$. Man könnte an ein volksetymologisches -in-gale denken. In verdingale, vardingale von franz. vertugade (-ale), span. verdugado steckt wohl in der Tat die Präposition in, aber durch Vermischung mit einem andern gleichbed. und demselben Kulturkreis angehörigen Worte: span. guardainfante, ital. guardinfante. Der Gegenstand den das Wort bezeichnete, der Reifrock forderte geradezu Umdeutungen und Umbildungen heraus; so machten die Franzosen verdu- zu vertu- und fühlten -garde in -gade ("Tugendschutz"); in Anlehnung daran sagten die Engländer iardingard (Jam.) und auf eigene Hand farthingale. In popinjay (me.popingay, papengay) ist das $n$ sicher schon aufserenglisch; das ausl. $n$ von arab. babayān, mhd. papegân erzeugte ein $n$ in der vorhergehenden Silbe: *papengan und wurde dann an seiner ursprünglichen Stelle durch $l l$, $q$ oder $i$ ersetzt. Die Form mit innerem $n$ ist im Altfranz. Mndl. ${ }^{1}$ Mnd. ä. und mdl. Deutsch belegt. - Der labiale Nasal scheint im Englischen nicht wie anderswo, und zwar z. T. sehr häufig, eingeschaltet worden zu sein. - Jespersen ${ }_{2}$ vermutet dafs der Ausfall des $n$ in so vielen Wörtern oder vielmehr das daraus sich ergebende Schwanken zwischen der Aussprache mit und der ohne $n$ zu dem Einschub eines Nasals in andern Wörtern geführt habe. Aber wenn man seine Belege für den erstern Vorgang näher ansieht, so enthalten sie sämtlich einen Nasal und ebenso verhält es sich mit dem gröfseren Teil von Ritters gleichsinnigen Belegen. Beide Erscheinungen, die dissimilatorische und die assimilatorische mögen ja zusammenhängen wie Ebbe und Flut, ohne dafs sich sagen liefse, die eine sei früher als die andere, und keinesfalls will ich hier die Möglichkeit einer umgekehrten Aussprache ausgeschaltet wissen. Jespersen, hatte nur die Stelle bestimmt an welcher Nasaleinschub

1 Ist ä.-holl. pijpengael\} altfranz. pipegale "Schubkarren" mit seinem $n$ dem mndl. papengaai nachgelolgt oder hat es wie dieses ursprünglich auf $n$ geendigt? J. J. Salverda de Grave De Franse woorden in het Nederlands bucht dies Wort S. 371 ; er hat es in die Liste der Belege für den Nasaleinschub S. 290 nicht aufgenommen. 
eintritt: die mittlere schwachbetonte Silbe eines Dreisilbers deren erste Silbe den Hauptton trägt. Logeman sucht aus der Natur dieser Silbe, aus einer "laxity of articulation“, aus der „nasal quality of the neutral vowel" den Vorgang zu erklären. Auch Ritter und Luick gehen von dem rhythmischen Typus $\left(\stackrel{\prime}{-} \times \dot{x}\right.$, bezw. $\left.{ }^{\prime} \times \dot{x} \times\right)$ der betreffenden Wörter aus; sie meinen dafs der zum Ausfall neigende Mittelvokal einer Stütze bedurfte, aber erst Luick bemüht sich nachzuweisen warum $n$ zu diesem Zwecke verwendet wurde. Seine physiologische Begründung ist nicht völlig abzulehnen, aber sie befriedigt auch nicht völlig. Nasal + Konsonant soll die eigentlich zur Stärkung der Silbe berufene Geminata ersetzen; aber nach Luicks eigener Angabe kommen die Geminaten für den Beginn des Nasaleinschubs kaum mehr in Frage; wie haben wir uns also die von ihm angesetzten nixteg-gale, mesed-dža(r) als lebendige Sprechweisen vorzustellen? Immerhin gebe ich eine ähnliche Beteiligung des "physiologischen" Faktors in solchen Fällen zu, werden wir doch unten S. 87.9I sehen dafs gewisse griech. und roman. Mundarten $-d z-,-(d) d \check{z}-$ regelmäfsig, bes. nach haupttonigem Vokal in $-n(d) z-,-n(d) z$ - übergehen lassen. Nur nicht die ausschliefsliche und selbst nicht die vorherrschende Betätigung dieses Faktors gebe ich zu. Kurz, ich denke, es beruht der englische Nasaleinschub auf der Wirkung eines benachbarten Nasals oder auch eines der Stellung nach entsprechenden in einem assoziierten ähnlichen Worte, ist aber an eine bestimmte Art von Silbe - eine mittlere nachtonige - gebunden; die Beschaffenheit des folgenden Konsonanten kann den Vorgang fördern. - Horn spricht nun von „überschriftsprachlichen" Formen wie milintary, skelinton, die man nicht mit "schriftsprachlichen" wie nightingale, passenger hätte zusammenwerfen dürfen. Der Gegensatz zwischen beiden Ausdrücken ist schief, jener bezieht sich auf den Ursprung, dieser auf den Gebrauch. $\mathrm{Ob}$ milintary u. dgl. bei mundartlich Redenden entstehen die "sich hochenglisch ausdrücken wollen", wird im allgemeinen schwer festzustellen sein. Aber ich verneine es falls solche Formen ganz so aufzufassen sind wie d. visentieren u. dgl. Das ist nämlich Horns Meinung sowie dafs über die Auffassung der letztern kein Zweifel sein könne: "lamentieren wurde mundartlich $\mathrm{zu}$ lametieren; deshalb wurde zu visetieren, visitieren ein vermeintlich schriftsprachliches visentieren gestellt". Allein visentieren und ähnliche Verben sind einfach an präsentieren usw. angeglichen (s. oben S. 80); lametieren (mit dissimilierendem Schwunde des $n$ ) kommt gar nicht ins Spiel. Und mit passenger nach messenger verhält es sich im Grunde nicht anders.

Ganz andere Art und Ausdehnung zeigt der griechische Nasaleinschub; er tritt schon seit alter Zeit auf und befällt ebenso Erbwie Lehnwörter. Eigens und eingehend hat sich damit W. Schulze Kuhns Zeitschr. 33 ('95), 366 ff. (u. d. T. „Samstag“) beschäftigt ; sodann in weiterem Zusammenhang länger oder kürzer K. Dieterich Untersuch. zur Gesch. d. griech. Spr. von der hellenistischen Zeit bis zum 10. Jhrh. n. Chr. ' 98, S. 92 ff. 28I, A. Thumb Die griechische 
Sprache in Zeitalter des Hellenismus 'o I, S. 135 ff., P. Kretschmer Der heutige lesbische Dialekt '05, Sp. $170 \mathrm{ff}$. Eine umfassende und entscheidende Beurteilung der Frage liegt hier nirgends vor und das rührt wesentlich daher dafs sei es ausschliefslich, sei es vorzugsweise die älteren Belege schärfer ins Auge gefalst worden sind. Denn ganz abgesehen davon dals die inschriftlichen und handschriftlichen Formen in sich selbst keine Bürgschaft für die treue Wiedergabe der Aussprache tragen, kann man Schulze nicht wohl beipflichten wenn er sagt, die über zwei Jahrtausende sich erstreckenden Zeugnisse seien „doch nur die Einzelwirkungen einer und derselben in Griechenland an den verschiedensten Orten und $\mathrm{zu}$ den verschiedensten Zeiten in wesentlich gleicher Richtung tätig gewesenen lautgeschichtlichen Tendenz" (S. 385). Thumb räumt ein, es sei „eine einheitliche Erklärung für alle Fälle nicht notwendig anzunehmen", und zwar bezieht er sich dabei nur auf die alte Zeit. Die Hauptsache ist die Verschiedenheit zwischen den Bedingungen der altgriechischen und denen der neugriechischen Fälle, auf die Dieterich nachdrücklich hingewiesen hat. Mit der gründlichen Untersuchung der letztern muls man beginnen, um an der Hand der in einzelnen Formen oder in Formengruppen ersichtlichen Übereinstimmungen in eine immer fernere Vergangenheit zurückzugehen. Thumb macht Dieterich einen Vorwurf der mir ungerechtfertigt zu

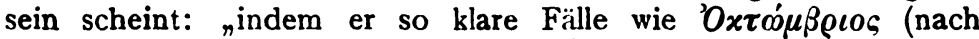
September, November, December) und $\lambda \dot{\eta} \mu \psi \varepsilon \sigma \vartheta \alpha \iota$ mit Verw. ${ }^{1}$ (nach $\lambda \alpha \mu \beta a ́ v \omega \infty)$ unter die übrigen einreiht, hat er den Sachverhalt verdunkelt ${ }^{\star}$. Der Sachverhalt konnte nicht verdunkelt werden, da er noch nicht im mindesten aufgehellt war, und wenn jene Fälle als klare von den übrigen als unklaren abgesondert würden, dann gewifs auch von manchen ganz gleichartigen, nur noch nicht als solchen erkannten, so von $\sigma v \mu \psi \varepsilon \dot{\lambda} \iota 0 \nu$, $\sigma v \mu \psi \varepsilon \iota \rho \iota x \dot{o}_{s}$, wo Thumb selbst die Einmischung von $\sigma v v$ - nicht erkannt hat. G. Meyer Neugr. St. III, 7 führt vier Beispiele von Nasaleinschub in lat. Lehnwörtern an; davon sind drei für mich Ergebnisse von Wortmischung. Bei zweien fällt sie auf griechischen Boden: $x \sigma^{\prime} \gamma \xi \alpha$ neben $x o^{\prime} \xi \alpha$ „Hüfte", "Kniekehle“, „Knöchel", $+x \dot{o}^{\prime} \gamma \chi \eta$ "Hirnschale“, „Augen-

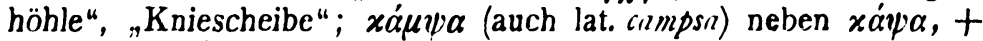
$x \dot{\alpha} \mu \pi \tau \varepsilon \iota \nu, x \dot{\alpha} \mu \pi \tau \rho \alpha$. Aber auf lateinischem Boden wurde flagellum, fragellum (+ frangere) $\mathrm{zu} *$ frangellum (kymr. fflangell; altlomb. franzelar); diesem entspricht $\varphi \rho \alpha \gamma \gamma \varepsilon \dot{\lambda}(\lambda) \iota-z u$ kühn dünkt es mich mit Schulze (S. 375 f.) aus dem häufigen einfachen $\lambda$ in diesem Worte auf ein $\gamma \gamma=g g$ als Vorstufe $z u$ schlielsen (vgl. Zeitschr. '10, $\left.279^{2}\right)$. Wie mit $\varphi \varrho \alpha \gamma \gamma \varepsilon \dot{\varepsilon} \lambda \lambda_{l}$ dürfte es sich mit

1 Vgl. altital. epilensia.

2 Hier steht aus Versehen: kymr. frangell. Ich muls annehmen dals flagellum und *frangellum im kymr. ffiangell zusammengetroffen sind; hier das $n g$, wie das Rhys und Loth tun, daraus zu erklären dals im Mittelkymr. zuweilen $g$ für $n g$ geschrieben wurde, scheint mir unzulässig. 


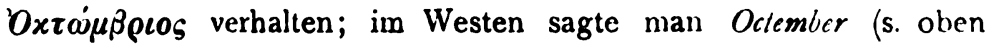
S. 72). Als breite Grundlage für den Nasaleinschub könnte man mit einiger Berechtigung den Nasalschwund ansehen, mit andern Worten jenen diesem gegenüber als umgekehrte Aussprache. Damit wäre aber wenig gewonnen; es bliebe uns keineswegs die Aufgabe erspart die näheren Bedingungen festzustellen unter denen der Einschub tatsächlich stattfindet; diese hätten die Geltung von eigentlichen Ursachen. Nach Thumb würde die Gegenwirkung des Nasalschwundes nur in die Schrift reichen; er fafst den Nasaleinschub vor Aspirata ebenso wie den vor Tenuis als umgekehrte Schreibung und zwar den letztern als Eigentümlichkeit der „notdürftig " hellenisierten Ägypter. Was den erstern anlangt, so sind die Fälle mit gedoppelter Aspirata besonders zu stellen. ${ }^{1}$ Leider

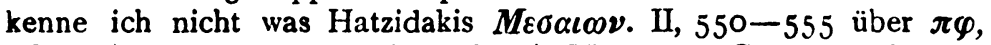
$\tau \vartheta, x \chi\{\mu \varphi, \nu \vartheta, \gamma \chi$ geschrieben hat (s. M. Vasmer Greko-Slawjanskie ètjudy III, 2 I f.); lebendige Formen bestätigen alte Schreibungen:

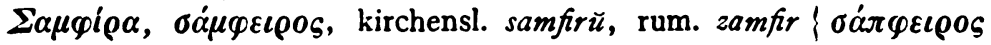
(denn es läfst sich schwer annehmen dafs hier das $n$ des altind. canipriyam fortdauere; das jüd.-aram. sampīiñnōn stammt aus dem

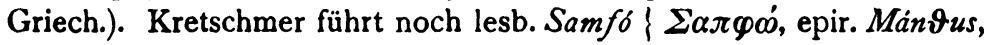

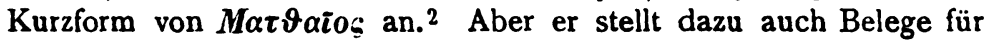
die gleichartige Vertretung gedoppelter Tenuis, inschriftliche Formen sowie noch heute gesprochenes $\beta \lambda \alpha \nu \tau i$ neben $\beta \lambda \alpha \tau \tau i$, Dem. von $\beta \lambda \alpha \dot{\tau} \tau \alpha$ \} blatla. Die Möglichkeit ist ja gegeben (s. oben S. 72) dafs eine doppelte Tenuis aus sich heraus einen Nasal entwickelt: $a t-t a\} a^{\prime}-t a\{a n-t a$, während ein $a$-ta unverändert bleibt; allein es muls bewiesen werden dafs kein anderer Einflufs dabei im Spiele ist." Einen solchen kann ich nicht umhin in dem auslautenden Nasal von $\beta \lambda \alpha \nu \tau i \nu$, der älteren Form von $\beta \lambda \alpha \nu \tau i$ zu erblicken (vgl.

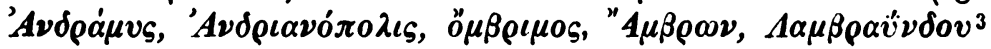

1 Aber bier wird das anl. $m$ gewirkt haben wie in $M \alpha \nu \vartheta \beta \omega \dot{\lambda} \lambda \varepsilon \iota 0 \iota\} M \alpha \vartheta \vartheta \alpha \beta$

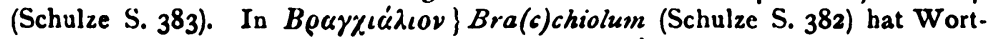

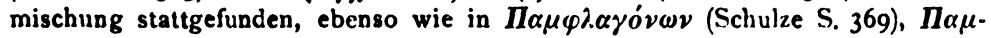
phayovlas (RC. R. Istit. Lomb. '10, 512 ).

2 Schulze S. 375, G. Meyer tolgend, nimmt an, zwischen Naviraxios,

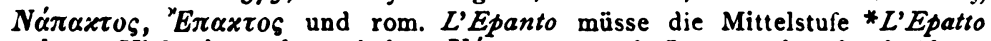
stehen. Vielmehr steht zwischen $N \alpha \pi \alpha \times \tau o s$ und Lepanto das durch ältere Karten und Portolane bezeugte Nepanto (K. Kretschmer Die ital. Portolane des Mittelalters S. 634); das $l$ - ist aus $n$ - ganz so dissimiliert wie z. B. in

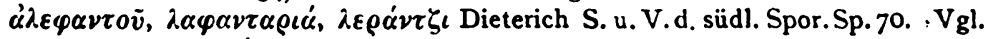
ital. Scarpanto \} Ká $\varrho \pi \alpha \vartheta o s$, was weder eine Geminata noch einen Nasal enthält; hier wirkte die Analogie andrer Ortsnamen auf -anto.

3 Der Nasaleinschub in diesem Worte erhält eine gewisse Bestätigung durch 2 wei davon und untereinander wohl unabhängige Formen: mail. lambarin altvic. slambarinto usw. und armen. lambiurint'os „Labyrinth". Ganz ähnlich gebaut ist prov. sembeli neben sebeli $(-n)$ "Zobelpelz $2^{4}$, zu dem wieder, von - $n$ und der Betonung abgesehen, lautlich slow. sembilja "Sibylle" stimmt. Hübschmann Arm. Etym. S. 331 gibt noch andere armen. Beispiele für einen entsprechenden Nasaleinschub in griech. Lehnwörtern: menḱ enay $=$ meḱenay $\} \mu \eta \chi \alpha \nu \eta^{\prime}$, 
bei Schulze; auch Semplumia ebd.), sowie in dem vorausgehenden

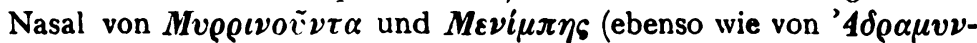
$\tau \eta v o ́ s\} ' A \delta \rho \alpha \mu v \tau \tau \eta v o ́ s$ bei Schulze). Die heutigen Mdd. sind hinsichtlich der Einwirkung eines benachbarten Nasals keine sichern

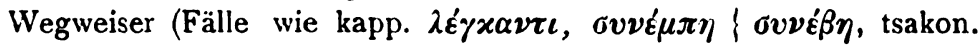

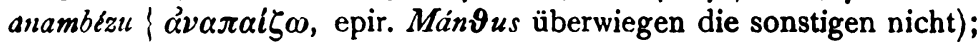
anderseits zeigen sie Nasaleinschub auch vor einfacher Tenuis. Ebenso vor $p s$ (auch $p t)$, so pont. $\lambda \varepsilon i \mu \psi \alpha \nu 0 \nu(m$ vor $n)$, $\boldsymbol{v} \mu \psi \propto \sigma \mu \alpha \nu(m$ vor $m)$,

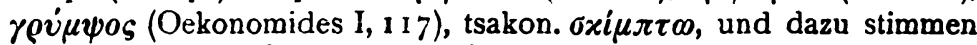
alte Formen wie $\alpha^{\prime} \mu \varepsilon i \mu \psi \psi \alpha \nu \tau \alpha, \alpha^{\prime} \nu \tau \dot{\alpha} \mu \varepsilon \iota \mu \psi \iota \varsigma, M \alpha \mu \psi o v x \rho \eta \dot{v} \eta, M o \mu-$

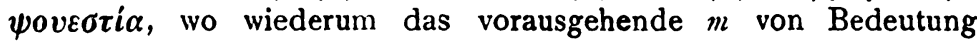
sein dürfte (vgl. Nempsis neben Nepsis Schulze S. 373). Thumb meint, die Griechen der hellenistischen Zeit hätten ebenso wie die heutigen eine Nedia nur in Verbindung mit Nasal gekannt, sodafs $\Sigma \alpha \mu$ -

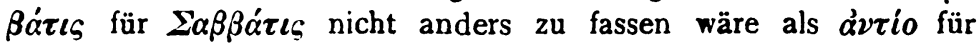
ital. addio, wo $\nu \tau$ für $d$ nicht nur geschrieben, sondern das $n$ darin auch mehr oder weniger deutlich gehört werde. Es würde hier also der Nasaleinschub einerseits auf dem Spirantischwerden der Media beruhen, anderseits auf dem Stimmhaftwerden der Tenuis nach $n$, wozu in manchen Mdd. noch der Ausfall des Nasals vor dem Verschlufslaut käme (diese Verhältnisse werden am besten veranschaulicht durch zwei lesbische Wörter die bei Kretschmer

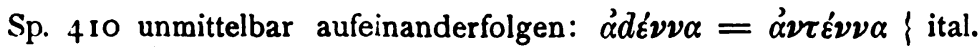
antenna; ádiov, in Ajasso ávdlov ; ital. addio). Aber der Nasal mochte auch schon in alter Zeit aus dem Blählaut der Media, besonders dem gedehnten hervorgehen, mit oder ohne Mitwirkung eines benachbarten Nasals. Das letztere geschieht in gewissen heutigen Mundarten. Als regelrechter Vorgang tritt uns da der Einschub eines $n$ vor (d) $d$ und $d z$ entgegen; so tsakon. sínda \} lak.

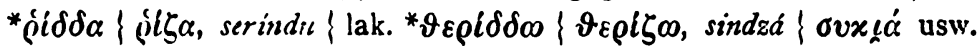

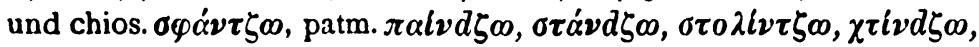
$x \rho \varepsilon \mu \alpha \dot{\alpha} d \zeta \varepsilon \iota, \beta v \nu d \zeta \alpha \dot{\nu} \varepsilon \iota, \rho i \nu d \zeta \iota, \mu \alpha \nu d \zeta i$, astyp. ler. $\pi \alpha i v d \zeta \infty$, $\varrho \imath \nu d \zeta \alpha$,

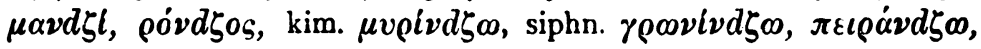

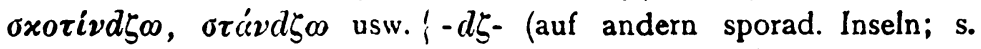
Dieterich Spr. u. Volksüb. der südl. Sporaden Sp. 58) $\{-\zeta-$. Vgl. dazu unten S. 91 das $-n \check{z}-\left\{-d d \check{z}-\right.$ einer oberital. Md. ${ }^{1}$

$k l m i n d r=k l m i d r\{x \varepsilon \rho c \mu i \varsigma$, simindr $\} \sigma \varepsilon \mu i \delta c i \lambda \iota s .-D a s$ von Schulze an-

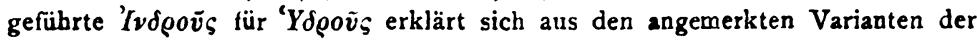
Kas. obl.: Hyndrunti, Indronto.

1 Erst in letzter Stunde kommt in meine Hände Man. A. Triandaphyllidis Die Lehnwörter der mittelgriechischen Vulgärliteratur '09; hier handelt $\S 31$ (S. 44-53) über die "Entfaltung eines vorkonsonantischen Nasals", bietet mir aber wenig Neues. Auch hier wird der Einfluls der von einem benachbarten Nasal ausgeht, vollständig übersehen (zu $\tau \rho \circ \pi \varepsilon \dot{\nu} \tau \alpha$ für $\tau \rho \circ \mu \pi \varepsilon \dot{\varepsilon} \alpha \mathrm{S} .47 \mathrm{vgl}$. slow. trobenta oben S. 76). Nur eines scheint mir, mit Bezug auf oben be- 
Für das Romanische scheint Foersters Panacee keinen Anklan: gefunden zu haben; was Meyer-Lübke im Grundrifs 1I, 534 gesagt hat: "Unklar ist die $\mathrm{N}$-Epenthese", wiederholt er ebenda ${ }^{2} \mathrm{I}, 68 \mathrm{I}$ (in seiner Rom. Gramm. I $\$ 587$ heifst es sogar: „Zu den schwierigsten Punkten gehört der Zutritt eines Nasals"). Aber an diesen Stellen sowie Rom. Gramm. I $\S 546$, Ital. Gramm. $\S 265$ führt er eine kalabrische Regel an, die ich als solche nicht gelten lassen kann. Und während ich sonst nicht die Absicht habe mich in romanische Einzelheiten zu vertiefen, mufs ich das hier doch tun weil man sich auf jene Regel zugunsten entsprechender Erklärungen in andern Sprachen berufen hat. Im Grundrifs 1.2 ist sie so geformt: „Nur im Kalabr. scheint Doppelkonsonanz zu Nasal + Kons. mit etwelcher Regelmälsigkeit $\mathrm{zu}$ werden: sumportare, landa $=$ latta, imbu gibbus mentu mitto u. a."; in der Rom. Gramm. I $\S 546$ so: „Eine eigentümliche Stellung nimmt das Kalabresische ein, sofern es nämlich gedehnten Konsonanten $n$ vorschlägt; vgl. mintivi $=$ mettere, mienzu $=$ mezzo, sunkurriri $=$ soccorrere, yimbu $=$ gibbo" $;$ in der Ital. Gramm. steht noch landa. Dieses ist ganz auszuscheiden (das $d$ für $t$ bliebe unerklärt); es deckt sich mit siz. lanna, welches wie auch bei Körting $\mathrm{zu}$ ersehen ist, lat. lamina entspricht. Während das Nordkalabrische $n n$ \} $n d$ hat wie das Siz., bewahrt das Mittel- und Südkal. $n d$; wie nun nordkal. siz. munnu usw. in dem zwischenliegenden Gebiete mundu usw. lautet, so hat man an die Stelle eines siz. lanna, nordkal. lannia (auch lanna?) ebenda ein landa, landia gesetzt, also einmal eine umgekehrte Aussprache hergestellt, während gleichartige umgekehrte Schreibungen ungemein häufig sind (Meyer-Lübke Ital. Gr. § 229). - Das $m b$ von jimbu, jumbu ist nicht blofs kalabrisch: siz. jimmu, tar. sciummo, neap. gimmo, gen. zembu, alttosk. gomba, ital. gomberuto. Es muls, wenigstens für Italien, ein frühes ${ }^{*}$ gymbus $\mid$ gybbus vorausgesetzt werden, an welchem altgr. $x i^{\prime} \mu \beta \eta$ "Ränzel“ (gr. von Bova kimbi „Buckel"; vgl. span. combo, -a, ital. gom[b]ito ${ }^{2}$ ) Anteil hat (griechischer Einfluls zeigt sich auch in ven. gufo "gekrümmt" ? $x v \varphi o ́ s)$; fördernd konnten andere begriffsähnliche Wörter mit derselben Konsonantenverbindung eingewirkt haben (so $\sigma x \alpha \mu \beta o ́ s$, ital. sghenzlio, mdl.-ital. sgalembo, lat. strambus neben strabus; vgl. auch span. zompo neben zopo u. a. Zeitschr. '91, 109). - In mienzu, mintere (die auch siz. sind; tar. miinzo, istr. menteva Ive S. I 30) hat ebenso

sprochene Gruppen, wichtig und gerade dieses dem Verf. unwichtig. S. 45 Anm. sagt er: „Ich erwähne nicht die Formen mit schwankendem $\tau \zeta: \nu \tau \zeta$, bei denen diese Konsonantenverbindung als approximative Wiedergabe des fremden

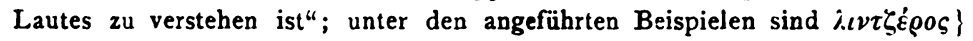

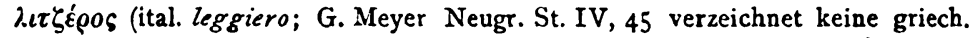

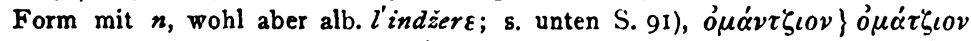
(ital. omaggio), $\left.\pi \alpha \sigma \dot{\alpha}^{\prime} \nu \tau \zeta o\right\} \pi \alpha \sigma \alpha^{\prime} \tau \zeta o$ anders zu beurteilen als $\left.\nu \tau \zeta \varepsilon \nu \varepsilon \rho \dot{\alpha} \lambda\right\}$ $\tau \zeta \varepsilon v \varepsilon \rho \alpha \lambda$ usw. Vielleicht beziehen sich hieranf die Worte: „In einigen Fällen wird der Nasal vom Lautkomplex $d z$ ausgelöst."

2 Vgl. oben S. 73. 
wie in miendula, mingránia, promintiu das $m$ - den Nasal hervorgerufen, nicht die Geminata, (vgl. ruzzu, váttcre) ${ }^{1}$; am stärksten hat sich diese Kraft des $m$ - im Port. entfaltet (Grundr. $\left.{ }^{2} I, 979\right)$ Mamburtii habe ich V. d. V. III, 206 aus einer römischen Inschrift des 6. Jhrh. n. Chr. angeführt. Der hier ausgesprochenen Ansicht hatte sich Meyer-Lübke anfangs (Ital. Gramm. $\$ 265$ ) zugeneigt.2 Kal, suncurrere, sumportare, sowie die andern Zusanmensetzungen mit sun- (sum-) \{ sub- (sumbarcare, sumbenire, sumbuzzare) sind nicht auf diese Mundart beschränkt; ihnen entsprechen südsard. sumbullai, suncirriri, sunfriri, log. sumbuzare und vor allem die "merkwürdigen" span. Formen mit son-(som-), zan-(zam-), wie sonreir, sampuzar (*subputtare ist also auf allen drei Gebieten vertreten; siz. sammuzzari stammt wohl aus dem Kal.). 3 Dieses sun- u. ä. erklärt sich sehr einfach aus sub-(su-, so-) + cum-, con- (co-, cu-), die ja beide im assimilierten Auslaut oft miteinander übereinstimmen (z. B. surridere, corrigere; *sullevare, colligere). Es sei auch an die häufige Verschmelzung anderer Verbalpräpositionen mit in- erinnert, z. B. prov. amparar, obw.-graub. anflar (ad- + in-), ansolver (ab- + in-), amblidar, unfrir (ob- + in-). Zudem finden manche begrifflichen Berührungen zwischen beiderlei Verben statt: ital. concorrere „beisteuern “ soccorrere, comportare = supportare "ertragen" 4 (in den kal. Texten bei Papanti wechseln, nach den Orten, beide miteinander). Schon in alter Zeit hat sich sub- dem cum- genähert; in meinem V.d. V. I, I14. 182 habe ich inschriftliches und handschriftliches sunbjunctas, sumiacente, sumditam angeführt und ebd. III, 93 dabei auf die span. son-Verben hingewiesen. In ähnlicher Weise hat in dem mehrfach aus alter Zeit belegten $\sigma v \mu \psi \varepsilon \dot{\lambda} \iota 0 \nu$ subsellium und $\sigma v \mu$ -

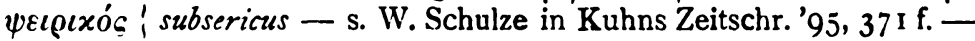

1 Keine Konstellation scheint für den Nasaleinschub günstiger zu sein als: m - dentaler Verschlufslaut vor Konsonant; zu diesem scheint der dentale Nasal die Brücke zu bilden vom labialen Nasal. Vgl. oben S. 75 Anm. 2 und Steindorff Koptische Gramm.2 \$ 56: „Die Einschiebung eines $n$ findet im $\therefore$ [aidischen] in der Silbe met, mĕt statt, die regelmälsig mnt geworden ist, i. B. somınt "drei" für *homet, mntre "Zeuge" für *mëtre" usw.

2 In matinu würde, wenn dem $n t$ ein $t$ zugrunde liegen mürste, das Nichteintreten der Nasalierung allerdings keiner Erklärung bedürfen, wohl aber einer solchen bei meiner Auffassung, und da glaube ich dals der zweifache Nasal hinderlich gewesen ist (wie bei engl. manager, s. oben S. 83; vgl. port. mai ãa, alt mani $\tilde{a} a$ ). Das Bretonische hat mintin neben mitin (wie mintr neben mitr, minut ueben mit); hier ist dem Nasaleinschub ein grö(serer Spielraum gewährt (s. V. Henry Lex. etym. S. 30 Anm. 4). - Ubrigens ist in südital. matinu nicht, wie Meyer-Lübke meint, $t$ aus $t t$ verkürzt; sondern matinu ist durch Silbenentdoppelung (ma[tu]tinum) entstanden, ital. mattino durch Vokalausfall (mat/u]tinum).

${ }^{3} \mathrm{Ob}$ hier südfranz. sanfloura $=$ soufloura, sufloura "défleurir", "déflorer", .écrémer" anzuschliefsen ist (s. Thomas Rom. '10, 186 Anm.), darüber bin ich noch zweifelhaft, nicht sowohl wegen der Vereinzeltheit als weil desenfloura, dessenfloura, dessanfloura (auch desafloura, dessefloura) als Nebenformen von desfloura vorkommen und dieses des-en- für des- im Südfranz. sehr häufig ist.

4 Vgl. unser vertragen = ertragen, wie mdl. vertrinken für ertrinken, verwelken für altes erwelken (also to verblühen) usw. 
der Grieche das fremde sub- in sein $\sigma v v$ - umgeformt. Die Anfügung des $n$ an $s u(b)$ - fand bis in ziemlich späte Zeit hinein statt; chapuzar dauert neben zampuzar fort, und das Altspanische kennt noch sosacar, sopesar = sonsacar, sompesar. Von den son-Verben ist mitgerissen worden sepelire, alt- und mdl.-ital. soppellire, kal. suppellire span. sobollir (alt), zabullir, zambullir; dazu kal. sepurcu, subburcu, sumburcu, semburcu (sanfrat. [Siz.] samuorch, griech. von Bova sempurtura Arch. glott. ital. IV, 80). Umgekehrt ist sin- mit su(b)- verwechselt worden in singultus (-tium): siz. kal. sugghiuzsu, kal. sugliuzzu, sugghiuttu usw.

Dafs der Sprachforscher um über einen einzigen Lautwandel einige Klarheit zu gewinnen, sich unzähliger Gänge unterziehen mufs, wird ihm die Laune nicht verderben; immerhin wird ihm ein allgemeines Unsicherheitsgefühl zurückbleiben. Er wird sich fragen: wie verhält sich denn ein solcher "sporadische" Lautwandel zum "Lautgesetz" ${ }^{\text {? }}$ stehen beide nicht im Widerspruch miteinander? Nun der Widerspruch ist nur scheinbar und wird einfach gelöst. Jener Wandel liegt nicht so tief, dieser nicht so hoch wie man meint; beide sind gesetzmälsig oder keiner. Die Wortgeschichte geht der Lautgeschichte voraus; diese löst sich aus jener ab und schwingt sich gebieterisch $z u$ ihrer Führerin auf; sie darf aber nicht vergessen dals sie in einer ganz bestimmten Abhängigkeit von ihr zu bleiben hat. Der Laut lebt nur im Worte und somit kommen bei irgendwelcher seiner Veränderungen alle die Bedingungen in Frage denen dieses untersteht. Ein Lautwandel ist zunächst im einzelnen Falle zu betrachten; es sind alle Umstände unter denen er sich vollzieht, festzustellen. Sie sind sehr mannigfacher Art, aber diese Arten stehen in inniger Berührung miteinander und es geht nicht an hier schroffe Abgrenzungen vorzunehmen, am wenigsten eine Zweiteilung auf Grund deren die rein lautlichen Erscheinungen allen andern gegenüberständen. Ziehen wir dann einen zweiten möglichst ähnlichen Fall heran, so werden wir finden dals er in einigen Umständen mit dem früheren nicht übereinstimmt; diese werden als unwesentliche beiseite geschoben. Dasselbe wiederholt sich bei jedem neuen Fall. Der Umfang eines Lautwandels wächst im umgekehrten Verhältnisse zu der Zahl der ihn bindenden Bedingungen. Zwischen dem Lautwandel der sich nur in einer Wortform aufzeigen lärst (so im port. fome \} fames; s. E. Wechssler Gibt es Lautgesetze? S. 143) und dem „ausnahmslosen“ besteht eine ununterbrochene Stufenreihe (und ebenso zwischen dem kombinatorischen und dem spontanen, wenn sie sich auch mit den andern beiden nicht völlig decken). In dieser Richtung, welche der tatsächlichen Entwicklung entspricht, ist der Weg leicht zu verfolgen; das Lautgesetz ist nichts Ursprüngliches, sondern etwas Gewachsenes. ${ }^{1}$

1 "Somwijlen hebben dergelijke sporadische klankovergangen zoo talrijk plaats, dat men van een onvolledige of halverweegsche klankwet zou kunnen spreken" B. Faddegon De alterneerende medeklinkers in het Indogermaansch 
Zur Erläuterung des Gesagten ist der Nasaleinschub vorzüglich geeignet. Auf romanischem Boden stellt sich jener vermeintlichen Regel des Kalabrischen ein von Meyer-Lübke Ital. Gramm. § 305 mit Recht als solches angesprochenes Gesetz einer tessinischen Mundart gegenüber. In der von Menzonio erscheint nämlich, zufolge Salvioni Arch. glott. ital. IX, 224, ganz regelmälsig $n \check{z}$ für (d)dž (ital. ǵǵ), z. B. manž maggio, ganža gaggia, mönž moggio, penž peggio, auch nach unbetontem Vokal. Er merkt an: "Mi sono per vero dettati, senza epentesi: müža muggire, ž $\bar{a} r \check{z}$ greggio, ma senza escludere che da altri si dica münž $\bar{a}$, žgrenž." Im übrigen Teil des von Salvioni durchforschten Landstriches kommen verhältnismälsig wenig Belege für diesen Lautwandel vor. Nicht durchgängig, aber doch in vielen Fällen tritt dieses $n \check{z}(n g)$ im Mail., besonders dem ländlichen ein (Salvioni Fonetica del dial. di Mil. $\S 263, b$ ), aber gerade nach $m$ - nicht: maǵg, möž, müǵǵdd. Einzelne Formen sind weitverbreitet, so lenger (linger) altgen. oberl.-graub. berg. usw. Es wäre sehr im allgemein-sprachwissenschaftlichen Interesse gelegen wenn das Gebiet um den Lago Maggiore und bis nach Mailand noch genauer mit Bezug auf diese Lauterscheinung untersucht würde, damit man sähe von welchen Ausgangspunkten, in welchen Richtungen sie sich ausgebreitet hat. Es ist leicht möglich dafs verschiedene Quellen zusammengeflossen sind. Meyer-Lübkes Vermutung dals mail. leng', renǵ, correnǵ [ital. -eggere] nach streng [ital. stringere] ge-

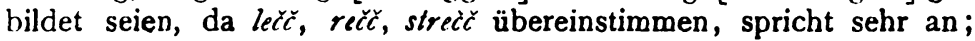
diese Formen begegnen uns auch in jener tess. Md. Jedenfalls aber spielt auch die Natur des folgenden $d \check{z}$, $\check{z}$ dabei eine ebenso bedeutsame Rolle wie auf andern Sprachgebieten (englisch, griechisch). 1 Ich schliefse mit einem Hinweis auf das sekundäre $n$ des Romanischen nach anlautendem Vokal ${ }^{2}$ und das von ihm sich abzweigende des Süditalienischen vor anlautendem Konsonanten, über deren Zugehörigkeit zu den rein lautlichen oder zu den analogischen Erscheinungen nur ein salomonisches Urteil entscheiden könnte. Ich sage das mit Bezug auf die Worte deren Ascoli am Schlusse seines tief erwogenen Aufsatzes über Formen vom Typus briaco, imbriaco sich bedient (Arch. III, 45I): „L' ultimo tipo della seconda categoria ( $\xi^{x}$-acquare en ${ }^{\zeta}$-aguare)... sempre $\dot{e}$ non altro che una spinta

(6ter Niederl. Philologenkongress 1910; SA.) S. If.). Vielleicht dürfte man auch von Lautgesetzchen reden; es handelt sich ja nicht um einen Wesens-, sondern um einen Gradunterschied.

1 Wie sich dies $n$ durch Verallgemeinerung der sonstigen Bedingungen ausdehnte, so auch durch die der wesentlichsten (vor (d) $\dot{z}$ ); nämlich es tritt auch vor stimmlosen dentalen Affrikaten und Spiranten auf, so mail. pincett, lansera (Salvioni Fon. mil. $§ 263, \mathrm{~b}$ ), berg. carensd, bellinz. cargans, margans (Salvioni Jahresb. I, 128), mod. verminzé, cadnanz, piem. nansa (Salvioni Zeitschr. '98, 477). Bemerkenswert ist istr. ngnse nozze (Ive 114. 130), dem istr.-rum. nunţe am nächsten liegt.

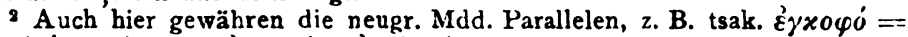

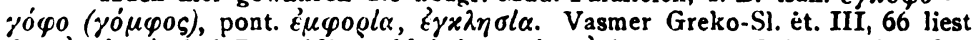
das $\varepsilon_{\gamma} \times \lambda \eta \sigma l \alpha$ bei Joannidis wohl irrigerweise $\varepsilon^{\prime} \lambda \eta \sigma \_\alpha$; anglisla, englisia hat auch das Gr. von Otranto (vgi, altrranz. englise). 
fonetica, e non mai un' operazione o trasformazione morfologica, che ci riporta in grembo alle serie in cui l' IN- è un elemento etimologico." Vielleicht würde er den gemischten Charakter des " in siz. ngranni, nfusku, kal. nguantu usw. haben gelten lassen wenn er, wie das nahe lag, seine Untersuchung hierauf ausgedehnt hätte. Diesen Fortgang hat Meyer-Lübke in der Ital. Gramm. $\$ 310$ vollzogen. Er sagt: „Es scheint nun auf den Gebieten wo in' zu nwird... der Anlaut $n$ Kons. so beliebt geworden zu sein dals er mehr und mehr an Stelle des einfachen Konsonanten tritt." Würde - was ja an sich denkbar ist - auf diesem Wege fortgeschritten, so käme man $\mathrm{zu}$ einem ausnahmslosen Lautgesetz: $n b-, n d-$ usw. ' $b$-, $d$ - usw., welches in manchen Sprachen sein Gegenstück fände ${ }^{1}$ und das doch aus einer Wortmischung erwachsen wäre. ${ }^{2}$ Allerdings hat wohl die Lautbeschaffenheit die Entwicklung gefördert und zwar werden wir dabei wieder an den Blählaut der Media denken, der ja gerade im Anlaut stark hervorzutreten liebt, besonders beim b: siz. bbeddu, bbonu, ǵǵeniu usw. (De Gregorio App. di fon. sic. S. 3o); man beachite die kal. Buchstabennamen (nach Accattatis): mbe (mme), nde (nne), nge, nzeta, aber cce, ppe, tte. Die Media aber konnte die mit gleicher Mundartikulation gesprochene und in manchen Fällen mit ihr wechselnde Tenuis anstecken; schliefslich konnten auch $f$ und $s$ die Nasalierungen annehmen.

1 So kann 2. B. im Tschaparek und im Guarani kein anl. $b, d, g$ ohne ein $n$ davor gesprochen werden.

2 Man halte es jenem "reinen Lautgesetz" gegenüber nach welchem jedes unmittelbar auslautende betonte $\dot{e}$ nach einem Nasal nasaliert wird, worüber ich in Kuhns Zeitschr. 20 ('72), 275 Anm. mich ausgelassen habe.

H. SCHUCHARDT. 